Ann. Sci. forest., I976, 33 (4), 257-269.

\title{
PRÉCISIONS DES MESURES DE SUPERFICIE ESTIMÉE PAR GRILLE DE POINTS OU INTERSECTIONS DE PARALLÈLES
}

\author{
R. B. CHEVROU \\ Inventaire Forestier National, \\ 1ter, avenue de Lowendal. \\ 75700 Paris \\ RÉSUMÉ
}

L'auteur présente des méthodes de calcul de l'erreur dans l'estimation de superficie par comptage de points, ou intersections de parallèles.

J. Bouchon a présenté (J. Bouchon, I975) une méthode permettant de calculer la précision de l'estimation d'une superficie par comptage de points.

De son côté, le service de l'inventaire forestier national a mis au point des méthodes, de type voisin, permettant de calculer simplement cette précision.

Cet article expose le cheminement théorique (1) qui a conduit aux formules utilisées par l'inventaire forestier.

\section{I. - PRÉSENTATION DE LA MÉTHODE E'T RÉSULTATS}

\section{I. - Estimation d'une superficie par la méthode des parallèles}

Soit une surface $\mathrm{S}$ délimitée par un périmètre $\mathrm{L}$.

Considérons un réseau fixe de parallèles équidistantes d'intervalle $a$, perpendiculaire à un axe $\mathrm{O} x$ que les parallèles du réseau coupent en des abscisses $k a$.

(1) L'approche de Matheron (G. Matheron, 1965) est différente et beaucoup plus générale : elle concerne aussi les volumes et les masses; notre article s'en tient aux superficies, et aboutit dans ce cas particulier à des calculs d'erreur plus simples. Il permet aussi la mise en relief des cas aberrants dont il faut s'écarter. 
Considérons un second réseau de parallèles équidistantes de même intervalle $a$, parallèle au premier réseau. Ce second réseau est mobile et il sert à estimer la superficie $s$ de $\mathrm{S}$ (fig. I).

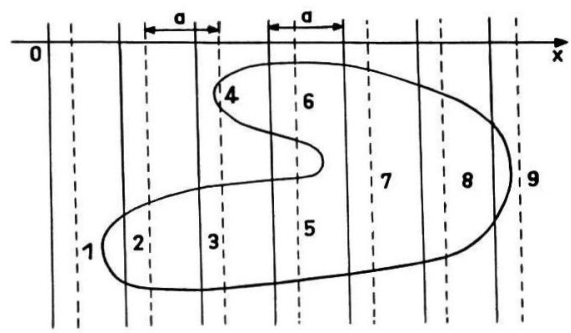

FIG. I. - Estimation d'une surface par la móthode des paralletes

Contimuous line $=$ fixed network; broken line $=$ mobile network

La surface $\mathrm{S}$, délimite sur ce second réseau, un maximum de $n$ segments de longueur égales respectivement à $l_{k}=f(x+k a), x$ variant entre o et $a$, et on peut estimer la superficie $s$ de $\mathrm{S}$ par :

$$
\hat{s}=a \sum_{k=1}^{n} f(x+k a)=a \sum l_{k},
$$

en limitant la somme aux $n$ termes utiles.

La superficie $s$ de $\mathrm{S}$ est égale à :

$$
s=\int f(x) d x=\sum_{k} \int_{k a}^{(k+\mathrm{I}) a} f(x) d x
$$

et l'espérance mathématique de $\hat{s}$, notée $\mathrm{E} \hat{s}$, est égale à $s$.

La variance de $\hat{s}$ peut être calculée comme suit :

$\hat{s}-s=a^{2} \sum f^{\prime}\left(k a+\xi_{k} a\right)$ où $\xi_{k}$ est compris entre o et $\mathrm{I}$,

$f^{\prime}$ étant la dérivée première de $f$

et

$$
\operatorname{var} \hat{s}=\mathrm{E}(\hat{s}-s)^{2}=a^{4} n g(n)
$$

où $n g(n)$ est une fonction dépendant, entre autre, de la variable $n$.

Cette fonction sera déterminée par la suite. Elle est écrite sous cette forme pour simplifier l'exposé ultérieur des calculs.

\section{2. - Estimation d'une superficie par la méthode de la grille de points}

Remplaçons maintenant les parallèles du réseau mobile par des lignes de points équidistants, d'intervalle constant $b$ (les points étant alignés dans 2 directions perpendiculaires).

Soit $\mathrm{N}$ le nombre des points intérieurs à $\mathrm{S}$.

On peut estimer $s$ par :

$$
\hat{s}=\mathrm{N} a b=a b \sum_{k=1}^{n} m_{k} \text { où } m_{k}=\text { nombre de points sur } l_{k} .
$$


Ia règle de la composition des variances permet d'écrire :

a)

$$
\operatorname{var} \hat{s}=a^{2} b^{2}\left\{\mathrm{E}\left(\operatorname{var} \Sigma m_{k}\right)+\operatorname{var}\left(\mathrm{E} \Sigma m_{k}\right)\right\}
$$

$$
\begin{aligned}
\mathrm{E} \Sigma m_{k} & =\mathrm{E}_{i}\left(\frac{\mathrm{I}}{b} \Sigma l_{k}\right) \\
\operatorname{var}\left(\mathrm{E} \Sigma m_{k}\right) & =\frac{a^{2}}{b^{2}} n g(n)
\end{aligned}
$$

b) On peut montrer, qu'en moyenne, il vient :

$$
\operatorname{var}\left(\Sigma m_{k}\right)=\Sigma\left(\operatorname{var} m_{k}\right) \text { (voir II ci-dessous). }
$$

Or var $m_{k}=p_{k}\left(\mathrm{I}-p_{k}\right)$ où $p_{k}$ est compris entre o et I et tel que :

$$
l_{k}=\left(m_{k}+p_{k}\right) b
$$

Si $p$ est uniformément réparti sur son intervalle de variation $(\mathrm{O}, \mathrm{I})$, la valeur moyenne de $p(\mathrm{r}-p)$ est égale à $\frac{\mathrm{I}}{6}$. On écrira donc :

$$
\mathrm{E}\left(\operatorname{var} \Sigma m_{k}\right)=\frac{n}{6}
$$

c) Il vient alors :

$$
\operatorname{var} \hat{s}=a^{2} b^{2} \frac{n}{6}+a^{4} n g(n)
$$

\section{3. - Valeur moyenne de $\mathrm{n}$}

Le nombre d'intersections du périmètre $\mathrm{L}$, dont la longueur est $l$, avec le réseau mobile de parallèles équidistantes est égal à $2 n$ et on sait que la valeur moyenne de ce nombre est liée à $l$ par la formule :

$$
\mathrm{E}(2 n)=\frac{2 l}{\pi a}
$$

done

$$
\mathrm{I}(n)=\frac{l}{\pi \sqrt{s}} \frac{\sqrt{s}}{a}=\frac{l}{\pi \sqrt{s}} \sqrt{\left(N^{\frac{b}{a}}\right)}
$$

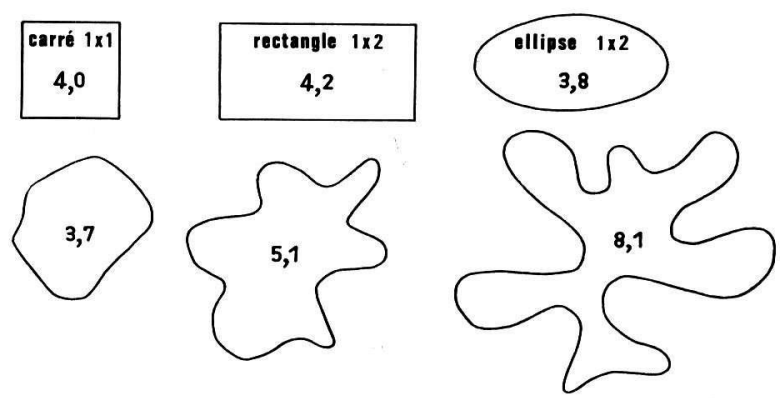

FIG. 2. - Coefficient de forme pour différentes figures 
$\frac{l}{\sqrt{s}}$ est un coefficient de forme, $\mathrm{C} f$, sans dimension qui ne dépend que des valeurs relatives de $l$ et de $\sqrt{ } s$. Il est facile de l'estimer sans même avoir à mesurer $l$ (voir fig. 2) car ce coefficient varie peu avec la forme. Pour plus de précision, $l$ peut être rapidement mesuré au curvimètre ou estimé à partir de $n$ par la formule $l=\pi a n$.

\section{4. - Variance de $\hat{s}$ dans la méthode de la grille de points}

La valeur moyenne de var $\hat{s}$ s'écrit alors, compte tenu des résultats précédents :

$$
\mathrm{E}(\operatorname{var} \hat{s})=\frac{a^{2} b^{2}}{\pi} \frac{l}{\sqrt{s}} \sqrt{\mathrm{N}}\left\{\frac{\mathrm{I}}{6} \sqrt{\frac{b}{a}}+\frac{a^{2}}{b^{2}} \sqrt{\frac{b}{a}} g(n)\right\}
$$

Posons $\lambda=\frac{b}{a}$

$$
\mathrm{E}(\operatorname{var} \hat{s})=\frac{a^{2} b^{2}}{\pi} \frac{l}{\sqrt{s}} \sqrt{\mathrm{N}}\left\{\frac{\sqrt{\lambda}}{6}+\frac{g(n)}{\sqrt{\lambda^{3}}}\right\}
$$

On peut intervertir les rôles de $a$ et de $b$ en considérant un réseau de parallèles perpendiculaires au premier, d'intervalle $b$, et sur ces parallèles des points équidistants d'intervalle $a$, sans modifier les positions des points les uns par rapport aux autres. On obtiendra une expression de var $\hat{s}$ de même forme que la précédente et qui doit lui être égale.

$$
\mathrm{E}(\operatorname{var} \hat{s})=\frac{a^{2} b^{2}}{\pi} \frac{l}{\sqrt{s}} \sqrt{\mathrm{N}}\left\{\frac{\mathrm{I}}{6 \sqrt{\lambda}}+\sqrt{\lambda^{3}} g(n)\right\}
$$

L'égalité de ces deux expressions donne $g(n)$ qui est inférieur ou égal à $\frac{\mathrm{I}}{\mathrm{I} 8}$ et que 1'on prend égal à cette valeur (égalité stricte pour $a=b) \cdot g(n)=\frac{\mathrm{I}}{\mathrm{I} 8}=0,055^{6}$

\section{5. - Variance et coefficient de variation de l'estimation $\hat{s}$}

De ce qui précède, il découle la valeur moyenne de la variance de $\hat{s}$.

a) Pour la méthode des parallèles équidistantes de $a$ :

$$
\mathrm{E}(\operatorname{var} \hat{s})=\frac{a^{3} l}{8 \pi}=0,0177 a^{3} l=0,0177 \frac{l}{\sqrt{s}} a^{3} \sqrt{s}=0,0177 \frac{l}{\sqrt{s}} a^{3} \sqrt{a \Sigma l_{k}}
$$

b) Pour la méthode de la grille de points systématique à maille carrée $a$ :

et

$$
\begin{aligned}
& \mathrm{E}(\operatorname{var} \hat{s})=\frac{2}{9 \pi} \frac{l}{\sqrt{s}} a^{4} \sqrt{\mathrm{N}}=0,0707 \frac{l}{\sqrt{s}} a^{4} \sqrt{\mathrm{N}} \\
& \mathrm{I}\left(\frac{\operatorname{var} \hat{s}}{s^{2}}\right)=\frac{2}{9 \pi} \frac{l}{\sqrt{ } s} \mathrm{~N}^{-3 / 2}=0,0707 \frac{l}{\sqrt{s}} \mathrm{~N}^{-3 / 2}
\end{aligned}
$$

\section{6. - Commentaires}

La variance de $\hat{s}$ ainsi calculée est une valeur moyenne. En fait, cette variance est très fluctuante, en particulier dans le cas où la surface $\mathrm{S}$ a un contour polygonal (voir plus loin le cas du rectangle). 
Il faut donc utiliser ces formules avec prudence.

Dans l'exemple traité par J. Bouchon, le périmètre de la surface est formé par 2 cercles, 1'un de rayon 5,64 cm, 1'autre de rayon $2,82 \mathrm{~cm}$ intérieur au premier. La longueur du périmètre de cette surface est égale à $53, \mathrm{I} 6 \mathrm{~cm}$; 1a surface est égale à $75 \mathrm{~cm}^{2}$; le coefficient de forme est donc égal à $6, \mathrm{I} 4$. La variance de l'estimation à l'aide d'une grille de points à maille carrée de I cm de côté est estimée égale à 3,59 par J. BouchoN (d'après la formule utilisée et les résultats expérimentaux). Ia formule développée ici donne une valeur de 3,76. Noter que ces valeurs sont indépendantes de la position relative du petit cercle à l'intérieur du grand cercle.

Une autre série de résultats expérimentaux provient de J. M. DELORD (Inventaire forestier national à Lyon). Les coefficients de forme sont égaux à 7,2 et I4,5 environ.

\begin{tabular}{|c|c|c|c|}
\hline \multirow{2}{*}{$\mathrm{C} f$} & \multirow{2}{*}{$n$} & \multicolumn{2}{|c|}{ Erreur relative $(\%)$} \\
\hline & & Observée & Calculée \\
\hline 7,2 & $\begin{array}{r}116 \\
30\end{array}$ & $\begin{array}{l}2,6 \\
4,4\end{array}$ & $\begin{array}{l}2,0 \\
5,6\end{array}$ \\
\hline 14,5 & 29 & 7,8 & 8,1 \\
\hline
\end{tabular}

F. LOETSCH et all. (I973) présentent une autre série de résultats expérimentaux obtenus avec des grilles à mailles carrées :

$$
\mathrm{C} f=4,67
$$

formule proposée par LoETSCH $=\log \mathrm{E} \%=\mathrm{I}, 782-0,76 \log n$ formule obtenue ici $\quad=\log \mathrm{E} \%=\mathrm{I}, 759-0,75 \log n$

avec $\mathrm{E} \%=$ erreur relative en $\%$

et $n=$ nombre de points intérieurs à la surface.

Les résultats suivants donnent $\mathrm{E} \%$ pour divers cas. La première valeur est celle observée par Lof'TSCH, la seconde est obtenue par les formules présentées ici.

\begin{tabular}{|c|c|c|c|}
\hline $\mathrm{C} f$ & $n$ & E\% observée & E\% calculée \\
\hline 6,0 & $\begin{array}{r}138 \\
70 \\
35\end{array}$ & $\begin{array}{l}1,2 \\
2,5 \\
3,3\end{array}$ & $\begin{array}{l}1,6 \\
2,7 \\
4,6\end{array}$ \\
\hline 6,5 & $\begin{array}{r}156 \\
79 \\
39\end{array}$ & $\begin{array}{l}1,2 \\
2,1 \\
5,0\end{array}$ & $\begin{array}{l}1,5 \\
2,5 \\
4,3\end{array}$ \\
\hline 6,8 & $\begin{array}{r}115 \\
59 \\
29\end{array}$ & $\begin{array}{l}1,2 \\
3,0 \\
4,2\end{array}$ & $\begin{array}{l}2,0 \\
3,3 \\
5,6\end{array}$ \\
\hline
\end{tabular}


LoETsch donne aussi quelques résultats obtenus avec une grille de parallèles placées perpendiculairement à la plus grande dimension de la surface.

L'intervalle est égal à $\mathrm{I} \mathrm{cm}$.

\begin{tabular}{c|c|c|c}
\hline \hline Périmètre $(\mathrm{cm})$ & $\mathrm{Cf}$ & $\mathrm{E} \%$ observée & $\mathrm{E} \%$ calculée \\
\hline 34,9 & 6,0 & 1,7 & 2,3 \\
\hline $40, \mathbf{t}$ & 6,5 & 1,4 & 2,2 \\
\hline 36,7 & 6,8 & 1,3 & 2,8 \\
\hline 47,2 & 4,7 & 0,4 & 0,9 \\
\hline \hline
\end{tabular}

On trouvera plus loin une explication de ces différences.

\section{II. - APPROCHE DIRECTE; DE LA VARIANCE}

I. - Retour à la formule générale

$$
\operatorname{var} \hat{s}=a^{2} b^{2}\left\{\mathrm{E}\left(\operatorname{var} \Sigma m_{k}\right)+\operatorname{var}\left(\mathrm{E} i \Sigma m_{k}\right)\right\}
$$

Cette expression est définie comme suit : E (var $\Sigma m_{k}$ ) est la valeur moyenne de la variance du nombre de points sur les lignes, et var $\left(\mathrm{E} \Sigma m_{k}\right)$ est la variance du nombre moyen des points sur les lignes, c'est-à-dire les longueurs interceptées par $\mathrm{S}$ sur les lignes parallèles à un coefficient près.

a) Le terme $E$ (var $\Sigma m_{k}$ ) est relativement stable en général.

En effet, considérons 2 parallèles quelconques, portant $m_{i}$ et $m_{j}$ points tels que $l_{i}=\left(m_{i}+p_{i}\right) b$ et $l_{j}=\left(m_{j}+p_{j}\right) b$.

Posons : $t_{1}=$ probabilité $\left(m_{i}\right.$ points sur $l_{i}$ et $m_{j}$ sur $\left.l_{j}\right)$

$t_{2}=$ probabilité $\left(m_{i}\right.$ sur $l_{i}$ et $\left.m_{j}+\mathrm{I} \operatorname{sur} l_{j}\right)$

$t_{3}=$ probabilité $\left(m_{i}+\right.$ I et $\left.m_{j}\right)$

$t_{4}=$ probabilité $\left(m_{i}+\mathrm{I}\right.$ et $\left.m_{j}+\mathrm{I}\right)$.

avec $t_{1}+t_{2}+t_{3}+t_{4}=\mathrm{I}, t_{3}+t_{4}=p_{i}$ et $t_{2}+t_{4}=p_{j}$.

Un calcul simple montre que :

Covariance $\left(m_{i}, m_{j}\right)=t_{1} t_{1}-t_{2} t_{3}$ dont la valeur moyenne est nulle.

On a donc, en général : $\mathrm{E}\left(\operatorname{var} \Sigma m_{k}\right)=\mathrm{E}\left(\Sigma\right.$ var $\left.m_{k}\right)=\frac{n}{6}$ comme dans le cas où les lignes de points sont indépendantes les unes des autres, les points étant alignés sur les lignes, mais non alignés dans le sens perpendiculaire aux lignes (forme de grille de points que l'on pourrait utiliser). 
On verra plus loin que les covariances peuvent être grandes, la variance de $\hat{s}$ prenant alors des valeurs très grandes, de l'ordre de $n^{2}$, mais ce cas ne se présente qu'avec une fréquence très faible en théorie.

b) Le terme var $\left(\mathrm{E}, \Sigma m_{k}\right)$, qui, à un coefficient près, est l'expression de la variance de $\hat{s}$ dans la méthode des parallèles, peut fluctuer de façon importante en fonction des sauts de la fonction $f(x)$ (voir ci-dessous).

On appelle saut, une variation rapide de cette fonction, par exemple dans le cas où elle s'écrit :

$$
f(x)=\text { constante }+\mathrm{A}(x-\Phi)^{\alpha}
$$

où $\Phi$ est une constante comprise entre o et $a$ et $\alpha$ compris entre o et I.

\section{2. - Méthodes des parallèles. Cas du cercle}

En reprenant les notations du $\S \mathrm{I}-\mathrm{I}$, on voit que dans chaque intervalle défini par le réseau fixe, la longueur $l_{k}$ du segment intérieur à $S$ sur la parallèle du réseau mobile compris dans cet intervalle, donne une estimation de la surface intérieure à $\mathrm{S}$ délimitée par les deux parallèles voisines du réseau fixe.

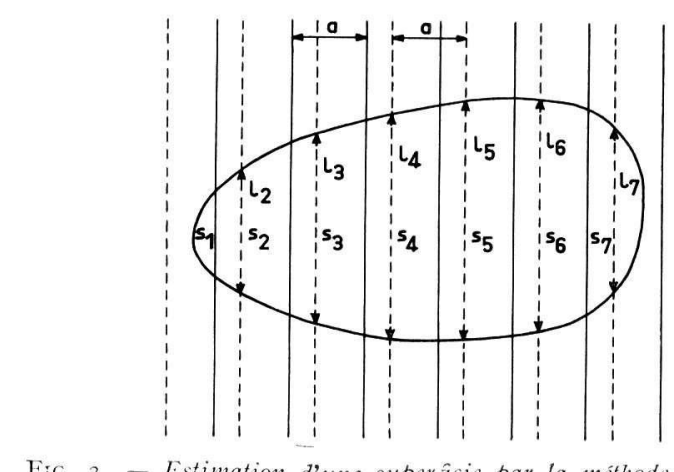

Fig. 3. - Estimation d'une superificie par la méthode des parallèles

Area estimation by transects

Avec des notations évidentes (voir fig. 3), on a :

et

$$
\mathrm{S}=s_{\mathbf{1}}+\sum_{k=2}^{n-1} s_{k}+s_{i}
$$

$$
\hat{s}=a l_{1}+a \Sigma l_{k}+a l_{n}
$$

On peut exprimer $l_{k}$ par un développement limité centré sur la valeur de $l$ au milieu de l'intervalle, sauf en présence d'un saut important.

Dans le cas général, l'expression de var $\hat{s}$ est complexe et suppose que la fonction $f(x)$ soit connue, au moins approximativement.

Dans le cas d'un cercle de diamètre D, les sauts ne se trouvent que dans le premier et le dernier intervalles et ils sont de la forme :

$$
2 \sqrt{a \mathrm{D}} \sqrt{ } \theta+\Phi \text { où } \theta=\frac{x}{a} \text { et } \Phi \text { compris entre o et } \frac{\mathrm{I}}{2}: 2 \Phi=\frac{\mathrm{D}}{a}-n
$$

ce qui est une approximation de l'équation du cercle. 
Dans ce qui suit, on suppose que le réseau fixe est placé de telle sorte que $s_{1}=$ $s_{n}$ et que, entre les milieux des $2^{\text {e }}$ et avant-dernier intervalles,

$$
\Sigma f^{\prime}=\int f^{\prime} \text { et } \Sigma f^{\prime \prime}=\int f^{\prime \prime} \text { où } f^{\prime} \text { et } f^{\prime \prime} \text { sont les dérivées de } f(x)
$$

En calculant $\mathrm{E}(\hat{s}-s)^{2}$, on obtient :

$$
\begin{gathered}
\operatorname{var} \hat{s}=\frac{a^{3} \sqrt{a \mathrm{D}}}{360}(\mathrm{I}+\Phi)^{-1}-\frac{8 a^{3} \mathrm{D}}{9}\left(\frac{\mathrm{I}}{2}+\Phi\right)^{3 / 2}(\mathrm{I}+\Phi)^{-1 / 2} \\
+\frac{32 a^{3} \mathrm{D}}{\mathrm{I} 5}\left(\frac{\mathrm{I}}{2}+\Phi\right)^{5 / 2}(\mathrm{I}+\Phi)^{-1 / 2}-\frac{\mathrm{I} 28}{\mathrm{IO} 5} a^{3} \mathrm{D}\left(\frac{\mathrm{I}}{2}+\Phi\right)^{7 / 2}(\mathrm{I}+\Phi)^{-1 / 2} \\
+4 a^{3} \mathrm{D}\left(\frac{\mathrm{I}}{2}+\Phi\right)^{2}-\frac{64}{9} a^{3} \mathrm{D}\left(\frac{\mathrm{I}}{2}+\Phi\right)^{3}+\pi a^{3} \mathrm{D} \Phi^{2}
\end{gathered}
$$

Les termes en $(\mathrm{I}+\Phi)$ représentent les dérivés de la fonction $f(x)$ au milieu des $2^{\mathrm{e}}$ et avant-dernier intervalles.

Ees valeurs prises par var $\hat{s}$ sont schématisées dans le graphique de la fig. 4 . On a porté sur le même graphique les valeurs prises par var $\hat{s}$ lorsqu'on supprime les termes $(\mathrm{I}+\Phi)$, ainsi que quelques valeurs obtenues par simulation approximative sur un calculateur de poche programmable.

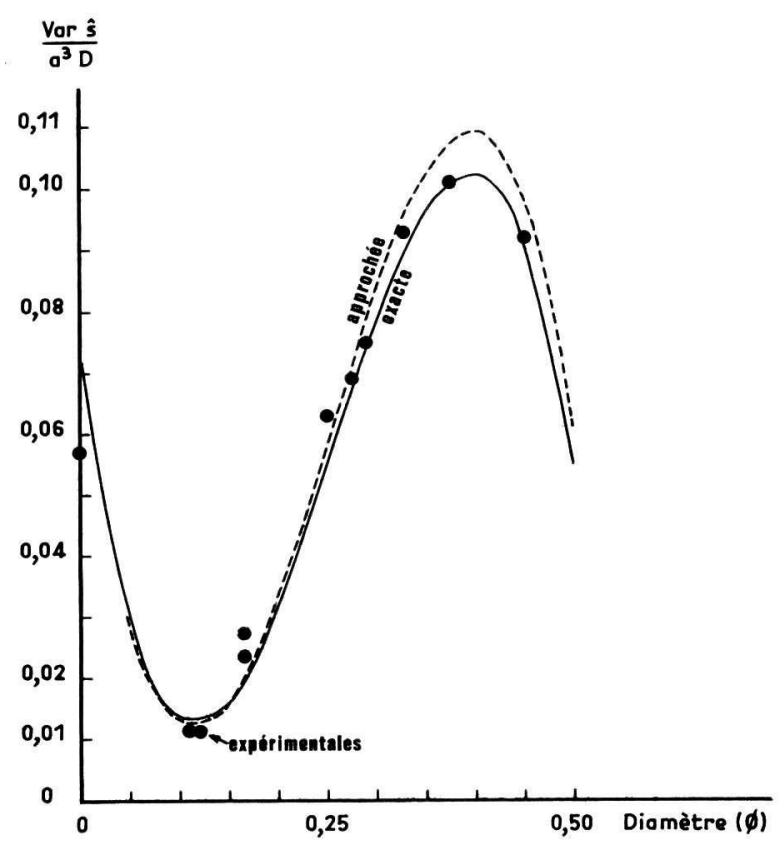

FIg. 4. - Variance s en fonction de $\Phi$ pour le cercle

Variance of area estimxtion $\hat{s}$ as a fonction of $\Phi$ for a circle

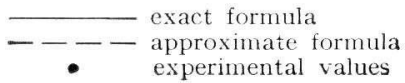


En supposant que $\Phi$ est distribué uniformément sur son intervalle de variation, la valeur moyenne de var $\hat{s}$ peut être calculée. Une intégration numérique donne:

$$
\mathrm{E}(\operatorname{var} \hat{s})=0,0576 a^{3} \mathrm{D}=0,0183 a^{3} l
$$

La suppression des termes $(\mathrm{I}+\Phi)$ suppose que les dérivées de la fonction $f$ dans les $2^{\text {e }}$ et avant dernier intervalles sont indépendantes de $\Phi$. Dans ce cas, la valeur moyenne de var $\hat{s}$ est :

$$
\mathrm{E}(\operatorname{var} \hat{s})=0,0608 a^{3} \mathrm{D}=0,0194 a^{3} l
$$

Le terme correspondant, donné par G. Matheron est :

$$
\mathrm{E}(\operatorname{var} \hat{s})=0,0609 a^{3} \mathrm{D}
$$

\section{3. - Méthode des parallèles. Cas général}

Il s'agit ici d'expliquer certaines méthodes parfois utilisées (voir F. YATEs, I960) en précisant les coefficients, encore que ces méthodes ne soient pas tout à fait satisfaisantes en pratique.

Ces méthodes consistent à prendre en compte les carrés des différences entre $l_{i}$ et $l_{i}+1$, la variance s'exprimant sous la forme :

$$
\operatorname{var} \hat{s}=\text { coefficient } x \sum_{i}\left(l_{i+1}-l_{i}\right)^{2}
$$

Si, dans un intervalle $i$, le saut est unique et de la forme :

$a \mathrm{R}_{i}\left(\frac{x}{a}-\Phi\right) \alpha$ avec $\alpha$ compris entre o et $\mathrm{I}$ et $\Phi$ entre o et $\mathrm{I}$, et si l'on place une parallèle au hasard dans cet intervalle, la part de la variance due à cette parallèle est égale à :

$$
\operatorname{var} \hat{s}_{i}=a^{4} \mathrm{R}_{i}^{2}\left\{\frac{(\mathrm{I}-\Phi)^{2 \alpha+1}}{2 \alpha+\mathrm{I}}-\frac{(\mathrm{I}-\Phi)^{2 \alpha+2}}{(\alpha+\mathrm{I})^{2}}\right\}
$$

La valeur du saut dans cet intervalle est :

$$
\Delta_{i}=a \mathrm{R}_{i}(\mathbf{I}-\Phi)^{\alpha}
$$

La valeur moyenne de $\frac{\operatorname{var} \hat{s}_{i}}{\Delta^{2}{ }_{i}}$ est égale à :

$$
\mathrm{E}\left\{\frac{\operatorname{var} \hat{s}_{i}}{\Delta^{2}{ }_{i}}\right\}=\left\{\frac{3 \alpha^{2}+2 \alpha+\mathrm{I}}{6(\alpha+\mathrm{I})^{2}(2 \alpha+\mathrm{I})}\right\} a^{2}
$$

Cette expression est égale à $\frac{a^{2}}{6}$ pour $\alpha=0$, $\frac{a^{2}}{\text { IO }}$ pour $\alpha=\frac{\mathrm{I}}{2}$ et $\frac{a^{2}}{\mathrm{I} 2}$ pour $\alpha=\mathrm{I}$.

En général les sauts sont de la forme où $\alpha=\frac{I}{2}$ ou $\alpha=I$ et on prendra donc:

$$
\operatorname{var} \hat{s}_{i}=\frac{\Delta^{2}{ }_{i} a^{2}}{\text { IO }} \text { et } \operatorname{var} \hat{s}=\frac{a^{2}}{\text { IO }} \sum_{i=1}^{n} \Delta^{2_{i}}
$$

$\Delta_{i}$ est pris égal à la différence entre 2 valeurs de $l_{i}$ consécutives avec $l_{o}=l_{n}+\mathrm{I}=0$. 
On prendra 6 au lieu de ro si le périmètre de S est en escalier, partout ou seulement localement.

Si les sauts sont à peu près également répartis dans tous les intervalles, $\Delta_{i}$ peut être estimé, en première approximation, par le rapport du diamètre apparent de $\mathrm{S}$, dans le sens perpendiculaire au réseau, divisé par la moitié du nombre d'intervalles utiles. Ceci explique pourquoi il y a intérêt, le plus souvent, à placer les parallèles perpendiculairement au grand axe de $S$.

L'erreur relative de l'estimation de $\hat{s}$ est égale à :

$$
\frac{\sqrt{\frac{\sum\left(l_{i+1}-l_{i}\right)^{2}}{I O}}}{\Sigma l_{i}}
$$

\section{4. - Méthode des parallèles. Cas particulier du rectangle}

Pour le rectangle, la valeur de var $\hat{s}$ s'exprime sous la forme $a^{3} l$ en général, sauf si 2 côtés sont parallèles au réseau de parallèles ou aux lignes de points.

Soit un rectangle de côtés $A$ et $B$ parallèles et perpendiculaires aux lignes de points et soit :

$$
\begin{aligned}
& \mathrm{A}=\left(n_{1}+p_{1}\right) a \text { avec } p_{1} \text { compris entre o et } \mathrm{I} \\
& \mathrm{B}=\left(m_{2}+p_{2}\right) b \text { avec } p_{2} \text { compris entre o et } \mathrm{I}
\end{aligned}
$$

a) Réseau de parallèles d'intervalle a :

$$
\begin{aligned}
& \hat{s}=\hat{n}_{1} \mathrm{~B} a \\
& s=\mathrm{E} i \hat{s}=\left(n_{1}+p_{1}\right) \mathrm{B} a \\
& \operatorname{var} \hat{s}=p_{1}\left(\mathrm{I}-p_{1}\right) \mathrm{B}^{2} a^{2} \leqslant \frac{\mathrm{I}}{4} \mathrm{~B}^{2} a^{2} \\
& \mathrm{E}, \operatorname{var} \hat{s}=\frac{\mathrm{I}}{6} \mathrm{~B}^{2} a^{2}=0, \mathrm{I} 67 \mathrm{~B}^{2} a^{2}
\end{aligned}
$$

On remarquera que $p_{1}$ est égal à $2 \Phi$ défini dans le cas du cercle ( $\$ 2$, ci-dessus).

b) Grille de points systématique d'intervalles a et b.

$$
\hat{s}=\mathrm{N} a b \text {. }
$$

Reprenons le raisonnement et les notations du $\S$ II. I $a)$ ci-dessus.

Nous avons ici $t_{2}=t_{3}=\mathrm{o}$ et $t_{1}=\mathrm{I}-t_{4}=p_{2}$ et donc $\operatorname{cov}\left(m_{i}, m_{j}\right)=p_{2}\left(\mathrm{I}-p_{2}\right)$.

Par conséquent, on peut écricre :

$\operatorname{var} \hat{s}=\mathrm{B}^{2} a^{2} p_{1}\left(\mathrm{I}-p_{1}\right)+\mathrm{A}^{2} b^{2} p_{2}\left(\mathrm{I}-p_{2}\right) \leqslant \frac{\mathrm{I}}{4}\left(\mathrm{~B}^{2} a^{2}+\mathrm{A}^{2} b^{2}\right)$

$\mathrm{E}(\operatorname{var} \hat{s})=\frac{\mathrm{I}}{6}\left(\mathrm{~B}^{2} a^{2}+\mathrm{A}^{2} b^{2}\right)$

Pour un carré et une grille à maille carrée, on a :

$$
\mathrm{E}(\operatorname{var} \hat{s})=0,02 \mathrm{I} a^{2} l^{2}=0,084 \frac{l}{\sqrt{ } s} a^{4} \mathrm{~N}
$$

c) Remarques.

En théorie, une telle position du rectangle par rapport à la grille ne peut se rencontrer qu'exceptionnellement, la probabilité pour qu'elle se présente étant infinitésimale. 
En pratique, cependant, cette position semble attirer l'opérateur. On doit donc imposer des règles pratiques interdisant l'apparition de cette position.

Si un seul côté de $\mathrm{S}$ est rectiligne, une autre solution consiste à imposer que ce côté soit placé à égale distance de 2 lignes successives, ou perpendiculaire aux parallèles du réseau.

\section{5. - Commentaires}

Les résultats ci-dessus et ceux obtenus par G. MATHERon (I965) montrent donc une certaine concordance entre eux et avec les résultats expérimentaux.

Il faut remarquer que var $\hat{s}$ peut fluctuer considérablement :

- avec $n$ (ou diamètre apparent de $\mathrm{S}$ dans le sens des lignes) ;

- avec les sauts du périmètre $L$, en fonction de l'orientation du réseau par rapport aux sauts ;

- avec le reste $(2 \Phi)$ de la division du diamètre apparent par l'intervalle du réseau (voir fig. 4).

I1 faut donc être prudent dans 1'estimation de $s$, et par conséquent définir des règles pratiques qui évitent qu'il n'y ait des sauts importants dans le sens des lignes (parallèles ou lignes de points).

\section{III. - GRILLE DE POINTS PSEUDO-SYSTÉMATIQUE}

\section{A MAILLE CARRÉE}

Considérons un double réseau de parallèles, le second étant perpendiculaire au premier, et de même intervalle $a$.

Supposons que, dans chaque carré défini par ce double réseau, on place un point au hasard.

Ces points forment une grille pseudo-systématique à maille carrée.

Plaçons cette grille sur la surface S (fig. 5).

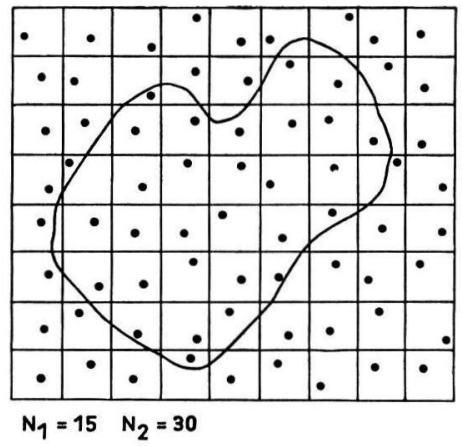

Fir. 5. - Grille de points pseudo-systématique à maille carrée 
$\mathrm{N}$ points tombent dans $\mathrm{S}$. La superficie $s$ de $\mathrm{S}$ est estimée par $\delta=\mathrm{N} a^{2}$.

Parmi les carrés contenus dans $S$ ou traversés par le périmètre $L$ de $S, N_{1}$ sont entièrement intérieurs à $\mathrm{S}$ et $2 \mathrm{~N}_{2}$ (à une unité près) sont traversés par L. Ėn moyenne, on a $\mathrm{N}=\mathrm{N}_{1}+\mathrm{N}_{2}$.

Dans un carré traversé par L, une superficie $p a^{2}$ (avec $p$ inférieur à I) est intérieure à $\mathrm{S}$ et la probabilité pour que le point de ce carré tombe dans $\mathrm{S}$ est égale à $p$, avec une variance égale à $p(\mathrm{I}-p)$ dont la valeur moyenne est $\frac{\mathrm{I}}{6}$.

Par conséquent :

$$
\operatorname{var} s=2 \mathrm{~N}_{2} a^{4} \frac{\mathrm{I}}{6}=\frac{\mathrm{I}}{3} \mathrm{~N}_{2} a^{4}
$$

Le périmètre $\mathrm{L}$ de $S$ entre dans un carré en traversant une ligne du double réseau. Le nombre de carrés traversés est, en moyenne, égal à $\mathrm{E}\left(2 \mathrm{~N}_{2}\right)$, soit :

$$
\mathrm{E}\left(2 \mathrm{~N}_{2}\right)=\frac{4 l}{\pi a}
$$

et par suite :

$$
\mathrm{E}(\operatorname{var} \hat{s})=\frac{2}{3 \pi} a^{3} l=0,2 \mathrm{I} 2 a^{3} l
$$

expression qui peut être réécrite sous la forme suivante :

ou encore :

$$
\mathrm{E}(\operatorname{var} s)=0,2 \mathrm{I} 2 \frac{l}{\sqrt{s}} a^{4} \sqrt{\mathrm{N}}
$$

$$
\mathrm{E}\left(\frac{\operatorname{var} \hat{s}}{s^{2}}\right)=0,2 \mathrm{I} 2 \frac{l}{\sqrt{s}} \mathrm{~N}^{-3 / 2}
$$

La variance de l'estimation obtente avec une grille pseudo-systématique à maille carrée est égale à 3 fois la variance de l'estimation obtenue avec une grille systématique de même maille.

Pur contre, la fluctuation de cette variance est beaucoup plus faible puisqu'elle ne dépend que de celle de $\mathrm{N}_{2}$ (ou $n$ ), ce qui signifie que l'utilisation d'une telle grille peut éviter des erreurs grossières.

Dans le cas du rectangle du paragraphe II-4, il vient approximativement : var $\hat{s}=0,167 a^{3} l$.

Alors qu'une grille systématique peut être construite avec un matériel réduit, ce qui permet de choisir une maille en rapport avec l'intensité du sondage désiré, une grille pseudo-systématique est plus difficile à construire puisque chaque point doit être placé au hasard dans le carré correspondant.

\section{CONCLUSION}

En notant $\mathrm{C} f$ le coefficient de forme, égal à la longueur $l$ du périmètre divisée par la racine carrée de la superficie $s$, les différentes formules donnant la variance de l'estimation $\hat{s}$ de $s$, s'écrivent : 
- Pour l'estimation par grille de parallèles d'intervalle $a$ :

$$
\text { var } \hat{s}=0,0177 a^{3} l=0,0177 \mathrm{C} f a^{3} \sqrt{\mathrm{s}}
$$

- Pour 1'estimation par grille de points systématique à maille carrée de côté $a:$

$$
\operatorname{var} \hat{s}=0,0707 \mathrm{Cf} a^{4} \sqrt{\mathrm{N}}=4 \times 0,0177 a^{3} l
$$

- Pour l'estimation par grille de points pseudo-systématique à maille carrée de côté $a$ :

$$
\operatorname{var} \hat{s}=0,2 \mathrm{I} 2 \mathrm{C} f a^{4} \sqrt{\mathrm{N}}=3 \times 0,0707 \mathrm{C} f a^{4} \sqrt{\mathrm{N}}=\mathrm{I} 2 \times 0,0177 a^{3} l
$$

Ces formules sont confirmées par l'expérience mais les règles pratiques d'application doivent être définies de telle manière à éviter les cas aberrants.

$$
\text { Rę̧u pour publication en mars } 1976 .
$$

\section{SUMMARY \\ ACCURACY OF AREA ESTIMATION BY THE DOT GRID METHOD AND BY TRANSECTS}

This paper presents a method of computing the accuracy of the area estimation by the dot grid method and by transects. $\mathrm{C} f$ being a form coefficient, equal to the length $l$ of the area perimeter divided by the square root ot the area $s$, different formulas are obtained, giving the variance of the area estimation $\hat{s}$ :

- with transects (parallel lines of interval a) :

$$
\operatorname{var} \hat{s}=0,0177 a^{3} l=0,0177 \mathrm{C} f a^{3} \sqrt{s}
$$

- with a dot grid : square grid of side a and $\mathrm{N}$ dots systematically allocated inside $s$ :

$$
\operatorname{var} \hat{s}=0,0707 \mathrm{C} f a^{4} \sqrt{\mathrm{N}}=4 \times 0,0177 a^{3} l
$$

- with a dot grid : square grid of side $a$ and $\mathrm{N}$ dots inside $s$, each dot being located at random in a square of side $a$ (pseudo-systematic dot grid) :

$$
\operatorname{var} \hat{s}=0,212 \mathrm{C} f a^{4} \sqrt{\mathrm{N}}=3 \times 0,0707 \mathrm{C} f a^{4} \sqrt{\mathrm{N}}
$$

These formulas are corroborated by experimental results from BouchoN (I975), LoETsCH (I973) and DELORD and theoretical results from Matheron (I965).

\section{RÉFÉRENCES BIBLIOGRAPHIQUES}

Bouchon J., 1975. Précision des mesures de superficie par comptage de points. Ann. Sci. forest., I $975,32(2)$, I 3 I-I 34.

Loetsch F. et al., 1973. Forest inventory, II, p. 33 à 35 , BLV Munchen.

Matheron G., I965. Les variables régionalisées et leur estimation, p. Io6 à Iro, Masson et Ci॰, I965.

YATES F., I960. Sampling methods for censuses and surveys. $3^{\mathrm{e}}$ éd. p. 229 à 233. Charles Griffin and Co., London. 